\title{
TRATAMIENTO DE LA ENDOMETRIOSIS I Y II: REVISIÓN DE LA LITERATURA
}

\section{A review of the literature concerning treating endometriosis I and II}

Ivonne Jeannette Díaz-Yamal, M.D. *, Alef Tsade Sanabria-Gaitán, M.D.*

Recibido: mayo 9/07 - Aceptado: septiembre 8/08

\section{RESUMEN}

Objetivo: el objetivo de este manuscrito es hacer una revisión de la literatura disponible que aborde el tratamiento de la endometriosis I y II para el manejo del dolor, de la infertilidad y como coadyuvante en las técnicas de reproducción asistida.

Metodología: se realizó una revisión de la literatura disponible en las bases de datos PubMed/Medline con énfasis en guías de manejo desde 1992 hasta 2007.

Resultados: en el tratamiento del dolor en endometriosis I y II, tanto el manejo médico como quirúrgico es efectivo. No hay diferencia entre los diversos tratamientos médicos en la disminución del dolor; su elección estará determinada por la edad, el deseo de fertilidad de la paciente y los efectos secundarios de los mismos. Para el tratamiento de la infertilidad asociada a endometriosis, el tratamiento médico no es efectivo, pero el manejo quirúrgico parece benéfico para los estadios I y II, eliminando los focos de la enfermedad.

Conclusión: se requieren más estudios aleatorios controlados, comparando su uso con placebo o sin tratamiento. La cirugía también ha demostrado mejoría, pero no existen estudios que comparen el manejo médico con el quirúrgico.

* Médico Ginecoobstetra Hospital Militar Central, Especialista en Endocrinología y Medicina Reproductiva, Docente Universidad Militar Nueva Granada. Bogotá, Colombia.

** Médico Ginecoobstetra Hospital Militar Central. Directora Científica Unidad de Fertilidad Clínica Marly. Bogotá, Colombia. Correo electrónico: alefsanabria@yahoo.com
Palabras clave: endometriosis leve, endometriosis mínima, tratamiento quirúrgico, tratamiento médico.

\section{SUMMARY}

Objective: this manuscript was aimed at reviewing the available literature concerning treatment for endometriosis I and II in managing pain, infertility and as coadjuvant in assisted-reproduction techniques. Methodology: the literature available in PubMed/Medline databases was reviewed, emphasising management-guidelines published between 1992 and 2007.

Results: endometriosis is usually defined as being the presence of endometrial functional tissue from the uterine cavity found growing on organs in other parts of the body. Its form of presentation changes from slight forms lacking adherence (I - II) to severe forms accompanied by retraction and fixation of peritoneal, ovary and tubaric surfaces (III - IV).

Endometriosis predominantly appears in females during their reproductive age, average age for onset of symptoms being 20,1 +/-6,8; occurring in all ethnic groups. Its prevalence is estimated as being $10 \%$; nevertheless, it is found in $40 \%$ to $60 \%$ of females consulting for pelvic pain and in 20\% to $30 \%$ of those who consult for infertility.

Conclusions: no difference was found between different medical treatments in terms of reducing pain, choice of treatment being determined by a 
patient's age, the desire for becoming fertile and the indirect effect of such. Nevertheless, more random controlled studies are required for comparing their use to placebo or without treatment. Surgery has also been demonstrated to induce improvement, but no studies were found comparing medical to surgical handling. Medical treatment of endometriosis-associated infertility was not seen to be effective; however, surgical treatment seemed to be beneficial for states I and II in eliminating disease foci.

Key words: mild endometriosis, minimal endometriosis, surgical treatment, medical treatment.

\section{INTRODUCCIÓN}

La endometriosis es definida como la presencia de tejido endometrial funcional fuera de la cavidad uterina. ${ }^{1}$ Se localiza generalmente en la pelvis, frecuentemente en peritoneo, ligamentos uterosacros, ovarios y fondo de saco posterior.

Se presenta predominantemente en mujeres en edad reproductiva, la edad promedio de inicio de los síntomas son los 20,1 +/- 6,8 años y se encuentra en todos los grupos étnicos. Su prevalencia es estimada en $10 \%$, sin embargo se encuentra en un 40 a $60 \%$ de las mujeres que consultan por dolor pélvico y en el 20 a 30\% de las que consultan por infertilidad. $^{2}$

Su etiología no ha sido esclarecida y se han propuesto varias teorías, siendo la más aceptada la menstruación retrógrada y la implantación de células endometriales que llevan a lesiones endometriósicas, ${ }^{3,4}$ sumada a alteraciones inmunológicas e inflamatorias y genéticas.

La triada clásica de presentación es dolor, dispareunia e infertilidad, aunque un tercio de las pacientes pueden cursar asintomáticas. ${ }^{5} \mathrm{La}$ clasificación más utilizada es la de la Sociedad Americana de Medicina Reproductiva ASRM, la cual la divide en cuatro estadios. Varía desde formas leves sin adherencias (I y II), hasta severas con retracción y fijación de la superficie peritoneal, tejido periovárico y peritubárico (III y IV). La utilidad de esta clasificación es limitada, ya que no hay correlación entre el estadio de la enfermedad y el grado de dolor o el pronóstico con el tratamiento. ${ }^{6,7}$

Se reconoce que el tratamiento de la endometriosis se debe enfocar al manejo del dolor y de la infertilidad. El dolor depende de la localización y profundidad de los implantes endometriósicos, siendo los más dolorosos los profundos en áreas de gran inervación. ${ }^{8}$ La infertilidad puede ser resultado de la distorsión anatómica pélvica causada por fibrosis y adherencias, sin embargo en las etapas I y II se puede presentar infertilidad, al parecer por la producción de una variedad de sustancias en los implantes o en el tejido que los rodea, como prostaglandinas, factores de crecimiento u otros factores embriotóxicos, alteración en el desarrollo folicular, disfunción ovulatoria, fertilización inadecuada, fagocitosis de los espermatozoides, implantación defectuosa, defectos de la fase lútea, inhibición de alteraciones inmunológicas; esta relación aún es motivo de controversia. ${ }^{5}$

Hay consenso en que la enfermedad recurre en aproximadamente un 5 a 20\% de las pacientes por año, hasta alcanzar un acumulativo de $40 \%$ a los cinco años. ${ }^{9}$

No hay un acuerdo sobre cuál es el tratamiento más eficaz en los estadios I y II de la endometriosis, algunos autores abogan por el tratamiento médico, mientras otros prefieren el tratamiento quirúrgico. Por lo tanto, el objetivo de este manuscrito es hacer una revisión de la literatura disponible que aborde el tratamiento de la endometriosis I y II para el manejo del dolor, de la infertilidad y como coadyuvante en las técnicas de reproducción asistida.

\section{METODOLOGÍA}

Se realizó una revisión de la literatura disponible en las bases de datos PubMed/Medline con énfasis en guías de manejo y revisiones sistemáticas desde 1992 hasta 2007. 


\section{TRATAMIENTO DEL DOLOR ASO- CIADO CON ENDOMETRIOSIS}

\section{Manejo médico}

Teniendo en cuenta que los estrógenos estimulan el crecimiento de la endometriosis, los tratamientos médicos se han enfocado en la alteración hormonal del ciclo menstrual, cancelando la función ovárica y alterando el crecimiento endometrial y, por lo tanto, el de los focos endometriósicos. Las terapias médicas disponibles actualmente son igualmente efectivas en controlar dismenorrea, dispareunia y dolor pélvico, y mejoran los puntajes de severidad en comparación con placebo.

a) Antiinflamatorios no esteroideos: son la primera línea de tratamiento en dismenorrea y dolor pélvico, los cuales disminuyen la producción de prostaglandinas en los implantes endometriósicos, demostrando ser efectivos en la dismenorrea primaria, (OR 7,91; IC 95\% 5,65 a 11,09). ${ }^{10} \mathrm{Sin}$ embargo, en la revisión de Cochrane por Allen, se incluyó un estudio que compara naproxeno con placebo, el cual no mostró ninguna evidencia de mejoría de dolor en pacientes con endometriosis (OR 3,27; IC 95\% 0,61 a 17,69). ${ }^{11}$

b) Anticonceptivos orales (ACO): a pesar de su uso frecuente en esta patología, hay pocos estudios al respecto. El metaanálisis de Cochrane por Moore, incluyó sólo un estudio que cumplió los criterios de inclusión. El estudio evaluó el uso de ACO vs. agonistas de GnRH. Se observó que los análogos de GnRH son más efectivos que los anticonceptivos orales en su uso convencional en mejorar la dismenorrea, ya que con éstos las pacientes entran en amenorrea. No se observaron diferencias significativas entre los dos grupos de tratamiento al final del tratamiento, en lo referente a dispareunia (OR 4,87; IC 95\% 0,96 a 24,65) o a dolor no menstrual (OR 0,93; IC $95 \% 0,25$ a 3,53). En el seguimiento a los seis meses postratamiento no se presentaron diferencias entre los dos medicamentos, en cuanto a dismenorrea (OR 0,48; IC 95\% 0,08 a 2,90), ni en dispareunia (OR 4,87; IC 95\% 0,96 a 24,65).
En general hay poca evidencia al respecto, por lo cual se requieren más estudios. ${ }^{12,13}$

c) Danazol: la observación de estados hiperandrogénicos que inducen atrofia endometrial ha llevado al uso de danazol, cuyo fin es producir atrofia de los implantes endometriósicos y, por lo tanto, mejorar el dolor.

Los estudios incluidos en el metaanálisis de Cochrane, mostraron mejoría significativa después de seis meses de tratamiento con danazol, comparado con placebo. La mejoría se presentó incluso seis meses después de terminar el tratamiento, con y sin cirugía. Se presentaron varias insuficiencias en los estudios disponibles, de forma que se requieren estudios clínicos aleatorios (RTCs) comparando danazol vs. placebo vs. otros medicamentos. ${ }^{14}$

d) Progestágenos y antiprogestágenos: las drogas progestacionales producen decidualización del tejido endometrial con eventual atrofia. La progestina más utilizada es la medroxyprogesterona. La dosis usada es 20 a $30 \mathrm{mg}$ diarios hasta por 6 meses, en Europa se han utilizado hasta 100 mg diarios con iguales efectos secundarios. No hay estudios que comprueben que una dosis sea mejor que otra. ${ }^{15}$

La gestrinona es un esteroide antiprogestacional que produce disminución en la concentración de receptores de estrógenos y progesterona, y disminución de la globulina transportadora de hormonas sexuales. Tiene efectos androgénicos y antiestrogénicos. En un estudio multicéntrico, aleatorio, doble ciego, fue tan eficaz como los agonistas de GnRH en el tratamiento del dolor pélvico. ${ }^{16}$

En los estudios realizados, los progestágenos y antiprogestágenos han demostrado la misma eficacia que los otros medicamentos usados en endometriosis. Los datos son limitados, ya que no hay estudios controlados con placebo. ${ }^{15}$

El dispositivo intrauterino con levonorgestrel es una alternativa en la administración de progestágenos, éste produce decidualización y aciclismo 
del tejido endometrial y endometriósico; al inhibir los factores de crecimiento endometriales, sus efectos sistémicos son mínimos. Su uso posquirúrgico en mujeres con endometriosis, tuvo una significante reducción en los períodos de dolor, comparado con los que recibieron agonistas GnRH (OR 0,14; IC 95\% 0,02 a 0,75). ${ }^{17}$ Sólo se incluyó un estudio RCTs en el metaanálisis de Cochrane, de tal forma que se requieren estudios para soportar estos hallazgos iniciales. ${ }^{18}$

e) Agonistas GnRH: disminuyen la secreción de FSH y LH, llevando a un hipogonadismo hipogonadotrófico; el estado hipoestrogénico produce atrofia endometrial y amenorrea. Su uso durante 3 ó 6 meses no ha demostrado diferencias en la recurrencia de dolor pélvico crónico. Desafortunadamente su uso por más de seis meses ha demostrado disminución en la densidad mineral ósea. ${ }^{19}$ Se indica iniciar terapia "add-back" con estrógenos y progestágenos en dosis para mujeres menopáusicas, con el fin de disminuir los efectos secundarios de los análogos; con sólo progesterona no es protectora, ya que después de seis meses de tratamiento, se comporta igual que el tratamiento con sólo análogos de Ngr. ${ }^{20,21}$

Los estudios que comparan el uso de agonistas de GnRH vs. danazol o gestrinona han demostrado disminución de la densidad ósea con los agonistas y aumento de la densidad con el danazol o la gestrinona. (Standardized Mean Difference SMD $-1,17$; IC 95\% -1,73 a -0,62). ${ }^{21}$

El uso de terapia "add-back" con los agonistas GnRH y el uso de éstos solos, demostraron igual eficacia en el manejo del dolor de la endometriosis que el uso de sólo agonistas, y disminuyó los síntomas secundarios. ${ }^{21}$

f) Inhibidores de aromatasa: el anastrazole disminuye los niveles de estrógenos en 1 a 10\% de los valores pretratamiento y disminuye los niveles de aromatasas. Con dosis de $1 \mathrm{mg}$ al día, ha demostrado disminución del dolor y disminución en el tamaño de las lesiones endometriósicas. No se dispone aún de estudios concluyentes. ${ }^{22}$

\section{Manejo quirúrgico}

El manejo quirúrgico tiene como objetivo remover los focos endometriósicos aparentes y restaurar las relaciones anatómicas normales. El acceso por laparotomía o laparoscopia no ha demostrado diferencias en cuanto a su eficacia, sin embargo el costo y tiempo quirúrgico es menor por laparoscopia. ${ }^{23} \mathrm{En}$ la revisión de Cochrane del manejo del dolor por vía quirúrgica, sólo se evaluó un estudio clínico RCT. Un total de 74 mujeres con endometriosis I, II y III se asignaron a laparoscopia quirúrgica con ablación de implantes, lisis de adherencias y ablación del nervio uterosacro vs. sólo laparoscopia diagnóstica. El dolor disminuyó substancialmente en la laparoscopia quirúrgica, $62 \%$ vs. $22 \%$ en la diagnóstica. ${ }^{24}$ En otro estudio RTC, el tratamiento quirúrgico demostró mejoría del 80\% de los síntomas, respecto a 32\% de las pacientes con laparoscopia diagnóstica solamente. El 90\% de las pacientes continuó hasta un año después con mejoría del dolor. ${ }^{25}$ La revisión sugiere que la cirugía laparoscópica es más efectiva que el manejo expectante en el tratamiento del dolor asociado a endometriosis (OR 4,97; IC 95\% $1,85$ a 13,39$).{ }^{26}$ Este estudio no analiza cada procedimiento quirúrgico de forma separada. Los datos de los estudios excluidos de esa revisión apoyan verdaderamente esta conclusión. En general, las pruebas sugieren que la cirugía laparoscópica resulta útil en el tratamiento del dolor pélvico asociado con la endometriosis. Debido a que las pruebas son limitadas, se necesitan ensayos controlados aleatorios adicionales, tanto de la cirugía en comparación con el manejo expectante, como de la cirugía en comparación con el tratamiento farmacológico. ${ }^{27} \mathrm{La}$ mayoría de cirujanos prefieren la escisión completa de la lesión o vaporización completa, dejando tejido normal. La electrocoagulación superficial con diatermia puede destruir la lesión activa pero puede dejar el tejido tratado y fibrosis de base. ${ }^{28}$

Otras formas de tratamiento quirúrgico del dolor asociado con endometriosis, involucra la interrupción neural de las vías de conducción del dolor y la neurectomía presacra, la cual demostró disminución 
en el dolor dismenorréico, pero no en otros dolores pélvicos en dos RTCs. ${ }^{29,30}$

\section{TRATAMIENTO DE LA INFERTILIDAD ASOCIADA CON ENDOMETRIOSIS \\ Manejo médico}

La mayoría de las terapias médicas usadas para tratar el dolor asociado con endometriosis, han sido también usadas para tratar la infertilidad asociada. En un metaanálisis de Cochrane con 16 RCTs, se incluyó seis estudios comparando supresores de ovulación vs. no tratamiento, o placebo y 10 estudios comparándolos con danazol. Los supresores de ovulación comparados fueron medroxyprogesterona, gestrinona, GnRHa y ACOs, los cuales demostraron ser iguales, pero sin que ninguno de ellos mejorara la fertilidad al compararlos con no tratamiento o placebo. El OR después de la supresión de la ovulación vs. placebo o ningún tratamiento fue de 0,74 (IC 95\% 0,48 a 1,15). A pesar de la utilización de diversos agentes anovulatorios, estos datos fueron estadísticamente homogéneos, por lo cual no se justifica su uso como tratamiento en pacientes con endometriosis y subfertilidad. ${ }^{31}$

\section{Manejo quirúrgico}

Es la primera elección en pacientes con subfertilidad. En mujeres cuya única causa de infertilidad era endometriosis I - II, la resección de implantes por laparoscopia o laparotomía mejoró la fertilidad al compararlos con tratamiento médico; con mejorías del 81\% con laparoscopia, 84\% con laparotomía y $54 \%$ con tratamiento médico. ${ }^{32}$

El metaanálisis de Cochrane incluyó dos estudios RCTs que evalúan la mejoría de infertilidad asociada con endometriosis, con la cirugía laparoscópica. En un estudio canadiense con 341 mujeres infértiles con estadio I y II de endometriosis, las pacientes se asignaron a laparoscopia, con y sin manejo quirúrgico. El número de pacientes que lograron un embarazo fue mayor en el grupo tratado quirúrgicamente, $31 \%$ vs. $18 \%$ del grupo sin tratamiento
$(p=0,006)(\mathrm{OR} 2,03 ; \mathrm{IC} 95 \%$ 1,28 a 3,2). El estudio encontró una mejoría significativa en el grupo tratado, pero la tasa de fecundidad mensual de 4,6\% continuó por debajo de la fecundidad normal. El otro, un estudio italiano similar con 101 mujeres, no demostró beneficio, la tasa de embarazo fue del $20 \%$ en el grupo tratado quirúrgicamente y $22 \%$ en el no quirúrgico al año de seguimiento (OR 0,76; IC 95\% 0,31 a 1,88). A pesar de estos resultados divergentes, cuando los estudios son combinados, se observa un beneficio significativo del tratamiento quirúrgico (OR 1,64; IC 95\% 1,05 a 2,5). Aunque la eficacia de la cirugía en la endometriosis temprana para manejo de infertilidad continúa inconstante, la evidencia actual puntualiza un aumento en la tasa de fertilidad con este tratamiento. ${ }^{33}$

Por ello, si se diagnostica endometriosis mínima o leve por laparoscopia, se recomienda hacer manejo quirúrgico, pero si no se logra el embarazo, las pacientes deben tratarse de la misma forma que parejas con infertilidad inexplicada. Se ha observado que la incidencia de infertilidad es menor que en las pacientes con infertilidad inexplicable. ${ }^{34}$

\section{Reproducción asistida}

La respuesta uterina alterada a las hormonas sexuales que se observa en la endometriosis tiene efectos en la fertilidad y el embarazo.

Una revisión sistemática con tres RCTs, mostró aumento de las tasas de embarazo en pacientes con endometriosis I y II, con inducción de la ovulación con gonadotropinas e inseminación intrauterina; de $28 \%$ vs. $8 \%$ sin inducción; RR 3,3 (IC 95\% 1,2 a 9,4). ${ }^{35}$ Otro no mostró diferencias entre inseminación intrauterina y manejo expectante, ya que las tasas de embarazo fueron 29 y $20 \%$ respectivamente; RR 1,46 (IC 95\% 0,5 a 4,0). ${ }^{36} \mathrm{Sin}$ embargo, cuando se combinaron los dos RCTs el RR fue de 2,3 (IC 95\% 1,1 a 4,6); y el tercer RCT de la revisión mostró mejores tasas de embarazo al realizar la inseminación intrauterina en ciclos alternativos de inducción, con gonadotropinas o sin ellas; $19 \%$ vs. $0 \% .^{37}$ 
De la anterior revisión queda en claro la recomendación de realizar la inseminación intrauterina en ciclos inducidos con gonadotropinas.

La literatura muestra que las mujeres con endometriosis en todos los grados tratadas con FIV o ICSI tienen tasas de embarazo inferior, en comparación con las mujeres sin endometriosis con infertilidad por factor tubárico. ${ }^{38}$ La revisión realizada por Cochrane incluye tres estudios en los que la mayoría de pacientes tienen endometriosis grado III - IV, afirmando el hecho de que el pretratamiento de estas pacientes con un agonista de la hormona liberadora de gonadotropina $(\mathrm{GnRH})$, durante al menos tres meses (y hasta seis meses) antes de la FIV o de la ICSI logra, al menos, cuadruplicar las probabilidades de embarazo clínico (OR 4,28; IC 95\% 2,00 a 9,15).

Aún no se puede determinar si este tratamiento es igualmente beneficioso para los estadios leves y graves de endometriosis, y si un tipo de agonista es superior a otro a partir de los estudios existentes. ${ }^{39}$

El manejo médico prequirúrgico o posquirúrgico no demostró diferencia estadísticamente significativa en la recurrencia de la enfermedad o en las tasas de embarazo. ${ }^{40}$

\section{CONCLUSIONES}

En el tratamiento del dolor en endometriosis, tanto el manejo médico como quirúrgico es efectivo. No hay diferencia entre los diferentes tratamientos médicos en la disminución del dolor. Su elección estará determinada por la edad, el deseo de fertilidad de la paciente y los efectos secundarios de los mismos; sin embargo, se requieren más estudios RCTs. La cirugía también ha demostrado mejoría, pero no existen estudios que comparen el manejo médico con el quirúrgico. El uso del dispositivo intrauterino con levonorgestrel ha demostrado mejoría del dolor posterior al tratamiento quirúrgico, teniendo como ventaja su uso prolongado.

En el tratamiento de la infertilidad asociada a endometriosis I - II, el tratamiento médico no es efectivo, el manejo quirúrgico parece benéfico; y si no se logra el embarazo, se trata como infertilidad de causa desconocida, primero con inseminación intrauterina en ciclos estimulados con gonadotropinas, y en última instancia, con fertilización in vitro (FIV).

\section{REFERENCIAS}

1. Olive D, Pritts E. Treatment of endometriosis. N Engl J Med 2001;345:266-75.

2. Viganò $\mathrm{P}$, Parazzini F, Somigliana E, Vercellini $\mathrm{P}$. Endometriosis: epidemiology and etiological factors. Best Pract Res Clin Obstet Gynaecol 2004;18:177200.

3. Sampson JA. Peritoneal endometriosis due to the menstrual dissemination of endometrial tissues into the peritoneal cavity. Am J Obstet Gynecol 1927;14:422-69.

4. Ajossa S, Mais V, Guerriero S, Paoletti AM, Caffiero A. The prevalence of endometriosis in premenopausal women undergoing gynecological surgery. Clin Exp Obstet Gynecol 1994;21:195-7.

5. Burns WN, Schenken RS. Pathophysiology of endometriosis-associated infertility. Clin Obstet Gynecol 1999;42:586-610.

6. D'Hooghe TM, Debrock S, Hill JA, Meuleman C. Endometriosis and subfertility: is the relationship resolved? Semin Reprod Med 2003;21:243-54.

7. Chapron C, Fauconnier A, Vieira M, Barakat H, Dousset B, Pansini V, et al. Anatomical distribution of deeply infiltrating endometriosis: surgical implications and proposition for a classification. Hum Reprod 2003;18:157-61.

8. The Practice Committee of the American Society for Reproductive Medicine. Treatment of pelvic pain associated with Endometriosis. Fertil Steril 2006;86: S18-27.

9. Fedele L, Bianchi S, Di Nola G, Candiani M, Busacca M, Vignali M. The recurrence of endometriosis. Ann N Y Acad Sci 1994;734:358-64.

10. Marjoribanks J, Proctor ML, Farquar C. Fármacos antiinflamatorios no esteroideos para la dismenorrea primaria. (Revisión Cochrane traducida). En: La Biblioteca Cochrane Plus, número 4, 2007. Oxford: Update Software Ltd.

11. Allen C, Hopewell S, Prentice A. Non-steroidal anti-inflammatory drugs for pain in women with endometriosis. Cochrane Database Systematic Rev 2005;(4):CD004753. 
12. Vercellini P, Trespidi L, Colombo A, Vendola N, Marchini M, Crosignani PG. A gonadotropin-releasing hormone agonist versus a low-dose oral contraceptive for pelvic pain associated with endometriosis. Fertil Steril 1993;60:75-9.

13. Davis L, Kennedy SS, Moore J, Prentice A. Modern combined oral contraceptives for pain associated with endometriosis. Cochrane Database Syst Rev 2007;(3): CD001019.

14. Selak V, Farquhar C, Prentice A, Singla A. Danazol for pelvic pain associated with endometriosis. Cochrane Database Syst Rev 2007;(4):CD000068.

15. Prentice A, Deary AJ, Bland E. Progestagens and antiprogestagens for pain associated with endometriosis. Cochrane Database Syst Rev 2000;(2):CD002122.

16. Gestrinone Italian Study Group. Gestrinone versus a gonadotropin-releasing hormone agonist for the treatment of pelvic pain associated with endometriosis: a multicenter, randomized, double-blind study. Fertil steril 1996;66:911-9.

17. Vercellini P, Frontino G, De Giorgi O, Aimi G. Zaina B, Crosignani PG. Comparison of a levonorgestrel-releasing intrauterine device versus expectant management after conservative surgery for symptomatic endometriosis: a pilot study. Fertil Steril 2003;80:305-9.

18. Abou-Setta AM, Al-Inany HG, Farquhar CM. Levonorgestrel-releasing intrauterine device (LNGIUD) for symptomatic endometriosis following surgery. Cochrane Database Syst Rev 2006;(4): CD005072.

19. Fukushima M. Changes in bone mineral content following hormone treatment for endometriosis. Int J Gynaecol Obstet 1995;50:S17-22.

20. Irahara M, Uemura H, Yasui T, Kinoshita K, Yamada M, Tezuka M, et al. Efficacy of every-other-day administration of conjugated equine estrogen and medroxyprogesterone acetate on gonadotrophin-releasing hormone agonists treatment in women with endometriosis. Gynecol Obstet Invest 2001;52:217-22.

21. Sagsveen M, Farmer JE, Prentice A, Breeze A. Gonadotrophin-releasing hormone analogues for endometriosis: bone mineral density. Cochrane Database Syst Rev 2003;(4):CD001297.

22. Amsterdam L, Gentry W, Jobanputra S, Wolf M, Rubin JD, Bulun SE. Anastrazole and oral contraceptives: a novel treatment for endometriosis. Fertil Steril 2005;84:300-4.
23. Crosignani PG, Vercellini P, Biffignandi F, Constantini W, Cortesi I, Imparato E. Laparoscopy versus laparotomy in conservative surgical treatment for severe endometriosis. Fertil Steril 1996;66:706-11.

24. Sutton CJ, Ewen SP, Whitelaw N, Haines P. Prospective, randomized, double-blind, controlled trial of laser laparoscopy in the treatment of pelvic pain associated with minimal, mild, and moderate endometriosis. Fertil Steril 1994;62:696-700.

25. Abbott J, Hawe J, Hunter D, Holmes M, Finn P, Garry R. Laparoscopic excision of endometriosis: a randomized, placebo-controlled trial. Fertil Steril 2004;82:878-84.

26. Jacobson TZ, Barlow DH, Garry R, Koninckx P. Laparoscopic surgery for pelvic pain associated with endometriosis. Cochrane Database Syst Rev 2001;(4): CD001300.

27. Sutton CJ, Pooley AS, Ewen SP, Haines P. Followup report on a randomized controlled trial of laser laparoscopy in the treatment of pelvic pain associated with minimal to moderate endometriosis. Fertil Steril 1997;68:1070-4.

28. Barton-Smith P, Ballard K, Kent ASH. Endometriosis: a general review and rationale for surgical therapy. Reviews in Gynaecological and Perinatal Practice 2006;6:168-76.

29. Sharp HT. Surgical management of pelvic pain. En: Blackwell RE, Olive DL, eds. Chronic pelvic pain: evaluation and management. New York: SpringerVerlag; 1998. p. 153-66.

30. Proctor ML, Latthe PM, Farquhar CM, Khan KS, Johnson NP. Surgical interruption of pelvic nerve pathways for primary and secondary dysmenorrhea. Cochrane Database Syst Rev 2005;(4):CD001896.

31. Hughes E, Brown J, Collins JJ, Farquhar C, Fedorkow DM, Vandekerckhone P. Ovulation suppression for endometriosis. Cochrane Database Syst Rev 2007;(3): CD000155.

32. Paulson JD, Asmar P, Saffan DS. Mild and moderate endometriosis. Comparison of treatment modalities for infertile couples. J Reprod Med 1991;36:151-5.

33. Jacobson TZ, Barlow DH, Koninckx PR, Olive D, Farquhar C. Laparoscopic surgery for subfertility associated with endometriosis. Cochrane Database Syst Rev 2002;(4):CD001398.

34. De Sutter P. Rational diagnosis and treatment in infertility. Best Pract Res Clin Obstet Gynaecol 2006;20:647-64. 
35. Tummon IS, Asher LJ, Martin JS, Tulandi T. Randomized controlled trial of superovulation and insemination for infertility associated with minimal or mild endometriosis. Fertil Steril 1997;68:8-12.

36. Fedele L, Bianchi S, Marchini M, Villa L, Brioschi D, Parazzini F. Superovulation with human menopausal gonadotropins in the treatment of infertility associated with minimal or mild endometriosis: a controlled randomized study. Fertil Steril 1992;58:28-31.

37. Nulsen JC, Walsh S, Dumez S, Metzger DA. A randomized and longitudinal study of human menopausal gonadotropin with intrauterine insemination in the treatment of infertility. Obstet Gynecol 1993;82:780-6.

38. Barnhart K, Dunsmoor-su R, Coutifaris C. Effect of endometriosis on in vitro fertilization. Fertil Steril 2002;77:1148-55.

39. Sallam HN, García-Velasco JA, Dias S, Arici A, Sallam HN. Long term pituitary down-regulation before in Vitro fertilization (IVF) for women with endometriosis. Cochrane Database Syst Rev 2006;(1):CD004635.

40. Yap C, Furness S, Farquhar C. Pre and post operative medical therapy for endometriosis surgery. Cochrane Database Syst Rev 2004;(3):CD003678. 